\title{
Numerical Verification of Scaling for Scattering from Fractons
}

\author{
Erich Stoll, Max Kolb, ${ }^{(a)}$ and Eric Courtens \\ IBM Research Division, Zurich Research Laboratory, CH-8803 Rüschlikon, Switzerland
}

(Received 9 December 1991)

\begin{abstract}
The single-length-scale postulate for fractons and the scaling hypothesis for the dynamic structure factor are confirmed for scalar elasticity on bond-percolation clusters. The strain exponent $\sigma$ is determined in two and three dimensions. Because of granularity and finite-size effects, these results cannot be obtained from site-percolation models for which the short-range structure fails to scale. Dipoleinduced-dipole scattering is particularly sensitive to granularity.

PACS numbers: $63.50 .+\mathrm{x}, 61.90 .+\mathrm{d}, 78.30 .-\mathrm{j}$
\end{abstract}

The vibrational eigenmodes of sufficiently random fractals are strongly localized excitations called fractons [1]. The initial work considered the scaling with frequency $\omega$ of their density of states $N(\omega) \propto \omega^{\tilde{d}-1}$, where $\tilde{d}$ is the spectral or fracton dimension, independent from the fractal dimension $D$ of the support. It also formulated the "single-length-scale postulate" (SLSP) that on fractons the physically relevant lengths, such as wavelength, scattering length, or localization length, all measure the "fracton size" $\lambda$ and scale like $\omega^{-\bar{d} / D}$. For scalar elastic models the vibrational problem maps onto anomalous diffusion [2], and the conjecture was made that $\tilde{d} \simeq \frac{4}{3}$ for percolation networks in any space dimension $d$ [1]. The latter was confirmed by simulations which also established that for tensorial elasticity other values of $\tilde{d}$ apply [3]. More recent numerical work bypassed the determination of individual eigenmodes [4] to study much larger systems [5]. Many theoretical predictions were confirmed by experiments on aerogels [6]. The possibility to observe scattering from real fractals, for example, Raman scattering in the fracton regime [7], called for further theoretical developments. Alexander proposed a scaling form for the dynamic structure factor $G(q, \omega)$, and introduced a new and independent exponent $\sigma$ related to the average coherent strain of fractons [8]. This work was recently refined, and extended to various lightscattering mechanisms, notably the dipole-induced-dipole (DID) contribution [9]. In a recent Letter, Montagna et $a l$. present simulations of $G(q, \omega)$ and of the associated DID-scattering intensity $I(\omega)$ for the scalar elastic sitepercolation (SP) model [10]. They observe that the wave vector $q_{\max }$ at the maximum of $\omega^{2} G(q, \omega)$ scales with $\omega$ like $\lambda^{-1}$, but their results lead them to challenge the SLSP and other scaling hypotheses. This contrasts with analytical work for a deterministic fractal where $G(q, \omega)$ does scale [11].

In this Letter, we present new simulation results mostly on bond- rather than site-percolation clusters. Scalar elasticity and the full diagonalization of the dynamical matrix are used. The latter guarantees access to individual eigenstates, which is essential to fully test the SLSP. However, it severely restricts the maximum system size. We show that only for bond percolation (BP) is the size sufficient to examine the scaling of structure re- lated properties. The SLSP is verified for different physical definitions of length [9], such as (i) a moment of the distance $\mathbf{r}$, as defined in Ref. [12]; (ii) the localization length $l_{a}$, whose definition is suggested by the Thouless criterion of localization [13], $l_{a}^{D} \equiv\left[\sum_{i}\left|\psi_{i}^{a}\right|^{2}\right]^{2} / \Sigma_{i}\left|\psi_{i}^{a}\right|^{4}$, where $\psi_{i}^{\alpha}$ is the wave function of fracton $\alpha$ on site $i$ and the sums extend over the entire volume; and (iii) the scattering length. Furthermore we test the scaling of $G(q, \omega)$ and obtain its asymptotic behavior for $q \omega^{-\dot{d} / D}$ $\ll 1$. Finally, we show that the DID-scattering intensity $I(\omega)$ is very different for SP and BP clusters, and that it scales.

In BP, all sites are initially occupied and the bonds are randomly filled up to the critical occupation $p=p_{c}(0.5$ in $2 \mathrm{D}$ and 0.24881 in 3D) [14]. A realization is kept if the largest cluster percolates. All other clusters-including disconnected single sites-are then removed. This contrasts with SP, where sites are randomly filled up to $p=p_{c}(0.59275$ in 2D and 0.3117 in 3D) [14], and bonds are placed between all occupied nearest-neighbor sites. The random filling produces a short-range granularity in SP, evident on visual inspection of the mass distribution. SP clusters show a large amount of randomly missing single sites. BP clusters have many masses on neighbor sites that are not directly connected. The SP granularity produces a white-noise component in the static structure factor $S(q)$ as shown in Fig. 1. Simulations of two vastly

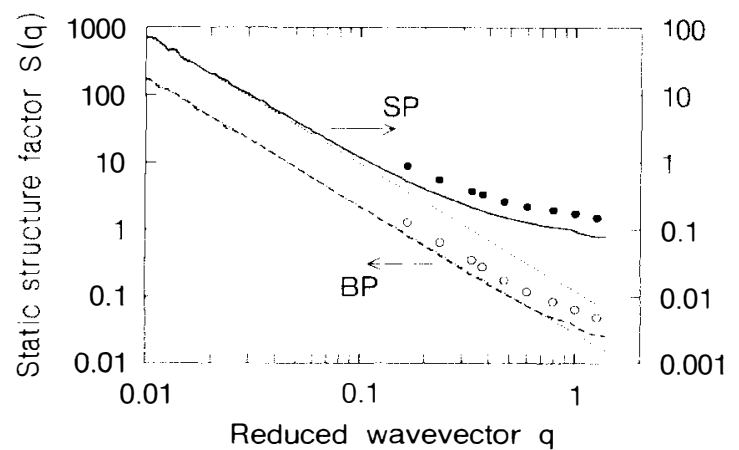

FIG. 1. The structure factor $S(q)$ for site-percolation (SP, top curve and solid dots) and bond-percolation (BP, bottom curve and open dots) clusters in 2D. The dots are for $L=12$ systems (averaged over 20000 realizations), and the full curves for $L=6800$. The dotted lines have the slope $-D=-\frac{91}{48}$. 


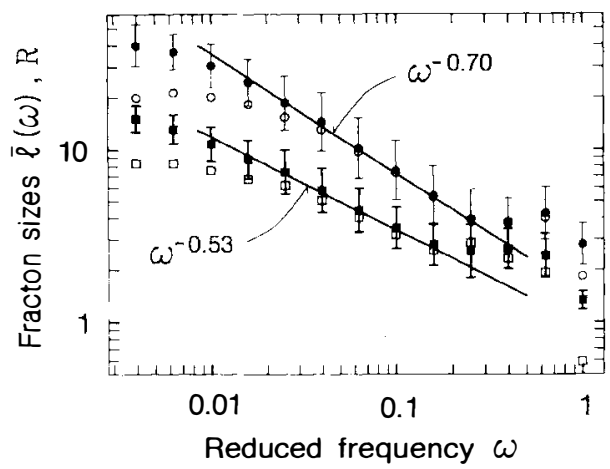

FIG. 2. The localization length $\bar{l}$ (solid symbols) and the mean radius $\mathcal{R}$ (open symbols) for BP fractons in two (circles) and three (squares) dimensions. The solid lines represent the expected scaling laws, $\lambda \propto \omega^{-\bar{d} / D}$. The vertical bars represent $\Delta \bar{l}$ as explained in the text.

different sizes $L$ are presented. The values of $q$ are in units of $\pi / a$, where $a$ is the unit cell size. The shape of $S(q)$ at high $q$ is $L$ independent, demonstrating that it is a true short-range effect. For BP, $S(q) \propto q^{-D}\left(D=\frac{91}{48}\right)$ already for $q<0.7$. For SP, the asymptotic regime is not quite reached for $q-0.05$. Because of universality, at sufficiently large sizes the two systems must obey the same scaling. However, because of the limit in the linear size $L$ of systems that can be diagonalized ( $L \$ 70$ or 30 , for 2D or 3D SP clusters, respectively) and since finite size starts playing a role for fractons at $\lambda \sim 0.4 L$ as shown below, we conclude that SP clusters are unsuited to determine structural properties of fracton eigenfunctions.

The SLSP is thus checked on percolating BP clusters. The equations of motion are standard [3,12]. Masses and spring constants for existing bonds are given the value 1 . The sizes are $L=68$ in 2D and $L=21$ in 3D, and the results are the average of 40 and 20 realizations, respectively. Frequencies are in units of the highest phonon frequency, $\omega_{\max }=\sqrt{4 d}$. First consider the localization length $\bar{l}$ in Fig. 2. This is the average of the $l_{\alpha}$ 's for all fractons of eigenvalue $\omega_{\alpha}$ in fixed-frequency intervals. The straight lines show the expected slopes, $-\tilde{d} / D$. The agreement with $\bar{l}$ in the middle-frequency range is excellent. On the high- $\omega$ side, deviations from the straight line occur for very small fractons, with $\bar{l} \leqslant 3$ in $3 \mathrm{D}$ and $\lesssim 4$ in 2D. On the low- $\omega$ side, the deviations occur for $\bar{l} \gtrsim 0.4 L$ because of finite-size effects. The vertical bars represent the root-mean-square deviation $\Delta \bar{l}$ of the $l_{\alpha}$ 's. The actual error on $\bar{l}$ is smaller than the size of the points. Remarkably, $\Delta \bar{l} / \bar{l}$ is approximately constant in the scaling region. Hence, the relative spread in fracton sizes is $\omega$ independent, which further supports the SLSP. The square root of the second moment, $\mathcal{R} \equiv\left[\left\langle(\mathbf{r}-\overline{\mathbf{r}})^{2}\right\rangle\right]^{1 / 2}$ - where $\overline{\mathbf{r}}$ is the center of gravity of the fractons and the angular brackets indicate averaging over the wave function [12]- is more sensitive to finite-size effects than $\bar{l}$, as it gives more weight to the periphery of the fractons. Hence, deviations of $\mathcal{R}$ from the scaling law are stronger at low $\omega$, as seen in Fig. 2. There remains to test the scattering length, and the wavelength $q_{\mathrm{max}}^{-1}$, and to this effect we consider now the scaling of the dynamic structure factor.

The dynamical density-density correlation function is defined by [9]

$$
G(\mathbf{q}, \omega)=\sum_{a} \delta\left(\omega-\omega_{a}\right)\left|\sum_{i} \mathbf{q} \cdot \mathbf{u}_{i}^{\alpha} e^{-i \mathbf{q} \cdot \mathbf{R}_{i}}\right|^{2},
$$

where $\mathbf{R}_{i}$ is the equilibrium position of particle $i$, and $\mathbf{u}_{i}^{\alpha}$ is the displacement produced by fracton $\alpha$. One has $u_{i}^{\alpha}=\left(k_{B} T\right)^{1 / 2} \psi_{i}^{\alpha} / \omega_{a}$, where $\boldsymbol{\psi}_{i}^{\alpha}$ is the normalized fracton eigenvector at site $i$ and $k_{B} T$ is the thermal energy. In the scalar case, $\left(\mathbf{q} \cdot \mathbf{u}_{i}^{\alpha}\right)^{2}$ is replaced by $q^{2}\left(u_{i}^{\alpha}\right)^{2}$, and the scaling ansatz is

$$
G(q, \omega)=\frac{k_{B} T}{\omega^{2}} g(q, \omega)=\frac{k_{B} T}{\omega^{2}} q^{y} h(x),
$$

with $x \equiv q \omega^{-\tilde{d} / D}$. The theory $[8,9]$ predicts that $y=2 \sigma$ $-D / \tilde{d}$, where $\sigma$ is the exponent relating the average displacement $u(\omega)$ to the average coherent fracton strain

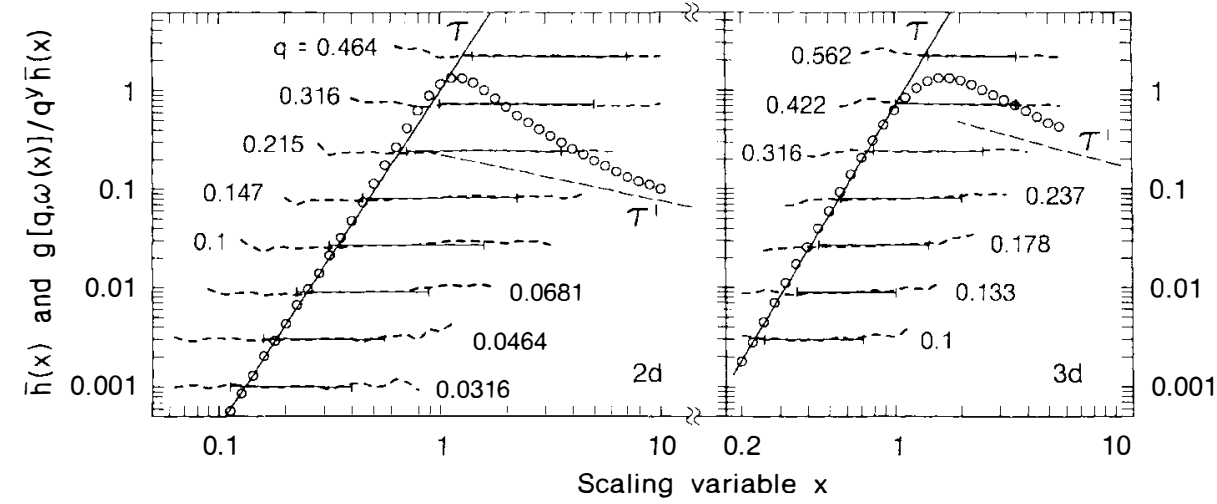

FIG. 3. The open circles are the scaling functions $\bar{h}(x)$ in 2D (left) and 3D (right). The asymptotes have the theoretical slopes $\tau$ and $\tau^{\prime}$. Also shown are the ratios $g(q, \omega) / q^{y} \bar{h}(x)$ for the indicated $q$ values. For clarity, these have been shifted vertically. They all are at the same scale, and in each case the ordinate of the horizontal bar is 1 . 
$\tilde{e}(\omega)=u(\omega) / \lambda^{\sigma}$. The $\omega$ dependence of the scaling function at fixed $q$ is derived from the eigenfunctions by calculating $\left|\sum_{i} \psi_{i}^{a} \exp \left(-i \mathbf{q} \cdot \mathbf{R}_{i}\right)\right|^{2}$. The results are summed within frequency intervals about each $\omega$ value. Using (1) and (2), one obtains $g(q, \omega)$. The index $y$ is then calculated from a least-squares fit of $g(q, \omega)=q^{y} h\left(q \omega^{-\bar{d} / D}\right)$, using for $\tilde{d} / D$ the standard values. The various curves $g(q, \omega) / q^{y}$ superpose so well that we rather show the mean scaling functions $\bar{h}(x)$ in Fig. 3. In addition we show separately the ratios $g(q, \omega) / q^{y} \bar{h}(x)$, together with the ranges used to calculate $\bar{h}(x)$ represented by horizontal bars at ordinate 1. The deviations of $g(q, \omega) / q^{y} \bar{h}(x)$ from 1 are seen to remain small well beyond the bars, and this at all $q$ 's. From $y$, using the above exponent relation, one finds $\sigma=1.05$ and 1.11 , in $2 \mathrm{D}$ and $3 \mathrm{D}$, respectively. This is compatible with the constraint [9] $\sigma \geq 1$, and with the intuitive notion that $\sigma$ is unlikely to be much larger than 1.

Considering the asymptotic behavior of $\bar{h}(x)$, we note that for $x \ll 1, q^{-1}$ is much larger than the fracton size. Then from (1) $g(q, \omega) \propto q^{4}$, and with $h(x) \propto x^{\tau}$, it follows that $\tau=4-2 \sigma+D / \tilde{d}$ [9]. These slopes (3.32 in 2D and 3.65 in 3D) are drawn in Fig. 3. The agreement with $\bar{h}(x)$ is remarkable, supporting the scaling hypothesis. For $x \gg 1$, one expects $h(x) \propto x^{-\tau^{\prime}}$. Now, $q^{-1}$ is smaller than the size of the relevant fractons. For each fracton different fractal "blobs," of size $\sim q^{-1}$, contribute separately to the scattering, both through their internal strain and by their overall motion. The internal strain dominates for $x \sim 1$, whereas intuitively one expects that the overall motions of small blobs control the limit $x \gg 1$. This is confirmed by the analysis of Ref. [9] which also gives $\tau^{\prime}=D-D / \tilde{d}$. Figure 3 suggests that our simulations do not reach this limit. However, the trend shown in Fig. 3-where the slopes are drawn with the theoretical $\tau^{\prime}$-are consistent with the above views [15].

The excellent scaling fit of $G(q, \omega)$ completes the test of the SLSP as it shows that the scattering length, as well as the wavelength $q_{\max }^{-1}$, scales like $\lambda$. This scaling is very sensitive to granularity. We attempted the same analysis for SP clusters, allowing in addition $\tilde{d} / D$ to vary, and observed that for them the scaling of $G(q, \omega)$ seriously fails.

We now turn to the DID-scattering intensities $I(\omega)$ calculated at $q=0$. For a fracton $\alpha, I^{a}$ is the square of a scattering amplitude $A^{\alpha} \propto \sum_{i, j}\left(\mathbf{u}_{i j}^{\alpha} \cdot \boldsymbol{\nabla}\right) \mathrm{T}\left(\mathbf{R}_{i j}\right) \cdot \mathbf{E}_{\text {app }}$ [9]. Here, $\mathbf{u}_{i j}^{a} \equiv \mathbf{u}_{i}^{\alpha}-\mathbf{u}_{j}^{\alpha}, \mathbf{R}_{i j} \equiv \mathbf{R}_{i}-\mathbf{R}_{j}, T_{m n}(r)=\left(3 r_{m} r_{n}\right.$ $\left.-r^{2} \delta_{m n}\right) / r^{5}$ is the dipole propagator where $m$ and $n$ are Cartesian indices, and $\mathbf{E}_{\text {app }}$ is the applied field. The result of this calculation we call the full- (F-) DID value. As the propagator $\mathbf{T}(r)$ decays rapidly with increasing $r$, one anticipates that DID scattering should be particularly sensitive to short-range granularity and bonding rules. For the same reason we also investigated partial sums in $A^{\alpha}$, over shells of fixed $R_{i j}$ [9]. The sum restricted to nearest neighbors, or NN-DID, is called short-range DID in Ref. [10]. Figure 4 shows the calculated Raman coupling coefficients, $C_{F}$ and $C_{\mathrm{NN}}$, respectively, where $C(\omega)$

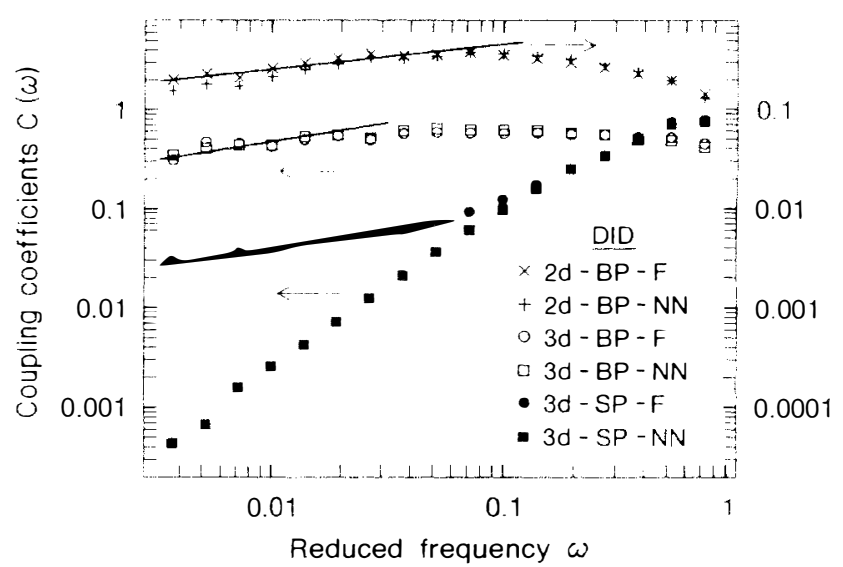

FIG. 4. The relative DID-coupling coefficients for the six cases indicated. The straight lines are drawn with the slopes from Eq. (3)

$\equiv \omega^{2} I(\omega) / N(\omega)$ is the mean scattering intensity per eigenmode. The calculation is for polarized scattering with polarization parallel to a principal lattice direction.

BP clusters are seen to scatter much more intensely than SP clusters, and with $C_{\mathrm{NN}} \simeq C_{\mathrm{F}}$ in both $2 \mathrm{D}$ and $3 \mathrm{D}$. This indicates a dominance of the $\mathrm{NN}$ contribution. It relates to the rapid decay of $T$, as confirmed by the partial sums decaying rapidly with increasing $R_{i j}$. The scaling derived from electrostatic considerations is [9]

$$
C_{\mathrm{F}}(\omega) \propto \omega^{(2 \dot{d} / D)(\sigma+d)-3 \dot{d}} .
$$

The straight lines in Fig. 4 are drawn with the corresponding slopes, 0.38 in $3 \mathrm{D}$ and 0.29 in $2 \mathrm{D}$. The lowfrequency BP data clearly support Eq. (3). The early departure from scaling as $\omega$ is increased presumably relates to short-range bonding rules. The SP behavior, shown here in 3D, is the average of 20 realizations with $L=29$. It is markedly different from BP. $C_{\mathrm{NN}}(\omega)$ is very steep, and $C_{\mathrm{F}}$ coincides with $C_{\mathrm{NN}}$ only at high $\omega$, crossing over to the scaling regime of Eq. (3) at low $\omega$. From partial sums on successive $R_{i j}$ shells, one finds that the low- $\omega$ behavior of the second-neighbor shell already parallels that of $C_{\mathrm{F}}$. In general the largest contributions to $C_{\mathrm{NN}}$ arise from $\mathrm{NN}$ masses loosely connected on the cluster, leading to large $u_{i j}$ 's. This only occurs in BP, while in SP NN masses are always directly connected and only at the highest $\omega$ can the $u_{i j}$ 's be large, owing to vibrations in antiphase. The direct bonding of all NN masses in SP also causes the rapid decrease of $C_{\mathrm{NN}}$ with decreasing $\omega$. In this case, the scaling regime can only occur by including interactions, propagating across missing sites, giving the crossover to the low-frequency scaling regime observed on $C_{\mathrm{F}}$. We also performed 2D SP simulations with $L=68$. In this case only a hint of a crossover remains in the F-DID calculation, suggesting that much larger simulations would be required for its observation. Considering the sums over separate shells at $R_{i j}$, one finds 
then that the scaling prediction of Eq. (3) is approximately recovered only from $R_{i j} \gtrsim 3 a$.

In conclusion, we demonstrated the validity of the single-length-scale postulate for vibrations on random fractals. Furthermore, we have shown that the structure factor scales according to the ideas of Alexander [8]. This does not require smooth eigenfunctions as stated in Ref. [10]. The light-scattering intensity scales as in Ref. [9]. The failure of a previous simulation is traced back to the short-range granularity affecting site percolation. More generally, the calculations suggest that structural properties in the embedding space are very sensitive to granularity. Bond percolation should always be preferred for simulations of fractal scattering properties.

The authors thank Professor S. Alexander and Professor R. Vacher for enlightening discussions. M.K. acknowledges the hospitality of IBM Research during a summer visit.

(a) Permanent address: Laboratoire de Chimie Théorique, École Normale Supérieure, F-69364 Lyon CEDEX 07, France.

[1] S. Alexander and R. Orbach, J. Phys. (Paris), Lett. 43, L625 (1982); R. Rammal and G. Toulouse, ibid. 44, L13 (1983).
[2] Y. Gefen, A. Aharony, and S. Alexander, Phys. Rev. Lett. 50, 77 (1983).

[3] G. S. Grest and I. Webman, J. Phys. (Paris), Lett. 45, L1155 (1984); I. Webman and G. S. Grest, Phys. Rev. B 31, 1689 (1985).

[4] M. L. Williams and H. J. Maris, Phys. Rev. B 31, 4508 (1985).

[5] E.g., K. Yakubo and T. Nakayama, Phys. Rev. B 40, 517 (1989).

[6] For reviews see, e.g., R. Vacher and E. Courtens, Phys. Scr. T29, 239 (1989); E. Courtens and R. Vacher, Philos. Mag. B (to be published).

[7] Y. Tsujimi, E. Courtens, J. Pelous, and R. Vacher, Phys. Rev. Lett. 60, 2757 (1988).

[8] S. Alexander, Phys. Rev. B 40, 7953 (1989).

[9] S. Alexander, E. Courtens, and R. Vacher (to be published).

[10] M. Montagna, O. Pilla, G. Viliani, V. Mazzacurati, G. Ruocco, and G. Signorelli, Phys. Rev. Lett. 65, 1136 (1990).

[11] U. Sivan, R. Blumenfeld, Y. Meir, and O. EntinWohlman, Europhys. Lett. 7, 249 (1988).

[12] E. Courtens, R. Vacher, and E. Stoll, Physica (Amsterdam) 38D, 41 (1989).

[13] D. J. Thouless, Phys. Rep. C13, 94 (1974).

[14] D. Stauffer, Introduction to Percolation Theory (Taylor and Francis, London, 1985); (private communication).

[15] This should be checked on large BP clusters, e.g., by calculating $G(q, \omega)$ from wave packets [4]. 